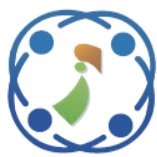

\title{
Efficient Dissemination of Rainfall Forecasting to Safeguard Farmers from Crop Failure Using Optimized Neural Network Model
}

\author{
Balamurali Ananthanarayanan $^{\text {1* }}$ Siva Balan $^{2}$ Anu Meera Balamurali ${ }^{3}$ Karthika Balamurali ${ }^{4}$ \\ ${ }^{I}$ Fishing Harbour Project Division, Fisheries Dept, Govt of Tamilnadu, India \\ ${ }^{2}$ Noorul Islam University, Tamilnadu, India \\ ${ }^{3}$ Anna university, Guindy campus, Chennai, India \\ ${ }^{4}$ Tata consultancy Services, Bangalore, Karnataka, India \\ * Corresponding author’s Email: balamuralia0765@gmail.com
}

\begin{abstract}
In the field of weather forecasting, especially in rainfall prediction many researchers employed different data mining techniques. There is numerous method of organizing agricultural engineering substance and it remains an open research issue particularly when taking to distinctive arrangements of clients - farmers, agricultural engineers, agri-organizations -both from proficiency point of view. Keeping these factors Indian farmers in mind, we have chosen to do research on efficient dissemination of rainfall forecasting to safeguard farmers from crop failure using optimized neural network (NN) model. Here, at first, we generate the feature matrix based on five feature indicator. Once the feature matrix is formed, the prediction is done based on the hybrid classifier. In hybrid classifier, particle swarm optimization algorithm is combined with Grey Wolf optimization for training the RBF NN. The performance of the algorithm is analyzed with the help of real datasets gathered from pechiparai and perunchani regions.
\end{abstract}

Keywords: Rainfall prediction, Hybrid classifier, Feature indicator, PSO, GWO, RBF, Neural network.

\section{Introduction}

Legitimate administration of water resources includes arranging, advancement and dissemination of water resources. These exercises are straightforwardly or in a roundabout way connected with the expectation of precipitation or spatial examination of the hydrologic cycle [1]. Among the key affecting parts of the hydrological cycle, precipitation forecast has an amazing part in spillover displaying and, thus, water resources administration [2]. A precise quantitative precipitation forecast can recognize the potential for substantial precipitation and conceivably related glimmer flooding and in addition giving data to hydrologic interests [3]. Likewise, precipitation bears different human biology co-operations, for example, horticulture, warm solace, and dissipation [4]. The presentations of both stochastic and deterministic precipitation forecast models [5] are influenced as precipitation is being a standout amongst the most convoluted segments of the hydrological cycle to forecast, and tremendous suspicious [6]. Numerous sorts of NN have been proposed for precipitation estimating, among which two broadly utilized classes are back engendering neural system (BPNN) [7] and outspread premise capacity neural system (RBF-NN) [8]. To overcome the drawback of BPNN, in [9] gray wolf optimizer (GWO) is hybrid to the BPNN. Moreover, the PSO algorithm is hybrid to FFNN to optimize the weight value [10]. To improve the predication accuracy, here we hybrid these two popular algorithm to select better connections to the network and the performance of the optimized network are to be measured in predicting future rainfall in a historical dataset. An accurate quantitative rainfall prediction can identify the potential for heavy rainfall and possible associated flash flooding, as well as providing information for hydrologic interests.

In this paper, we have developed an efficient dissemination of rainfall forecasting to safeguard farmers from crop failure using optimized neural network model. Firstly, the input rainfall dataset is 
preprocessed to make data suitable for extracting the feature indicators. Then, feature indicators are used to extract feature components from the preprocessed input data. Here, we used five types of feature indicator. Once the feature matrix is formed, the prediction is done based on the hybrid classifier. In the hybrid classifier, gray wolf optimization algorithm is combined with particle swarm optimization algorithm for training the RBF neural network. The hybridization approach increase the efficiency of the prediction performance compare to individual approach. The organization of this paper is as follows: a brief review of some of the literature works in rainfall prediction models are presented in Section 2. The proposed rainfall prediction model is detailed in Section 3. The experimental results and performance evaluation discussion are provided in Section 4. Finally, the conclusions are summed up in Section 5.

\section{Related Works}

Many of the researchers have explained the rainfall prediction approach. Among them some of the research papers explained in this section; Abdusselam and T. Assefa [11] have clarified the two strategies called consolidated season-multilayer perceptron and half and half wavelet-seasonmultilayer perceptron were produced to upgrade forecast precision and expand expectation lead time of day by day precipitation up to 5 days by utilizing information from two stations as a part of Turkey. Moreover, J. Wu et al. [12] have clarified the advancing RBF neural systems for precipitation prediction utilizing hybrid particle swarm optimization and genetic algorithm. Here, hybrid developmental calculations were utilized to naturally assemble a radial basis function neural networks (RBF-NN) that tackle a predefined issue, identified with precipitation determining for this situation. Additionally, R. Hashim et al. [13] have clarified the Selection of meteorological parameters influencing rainfall prediction utilizing the neurofuzzy system. Similarly, Indrabayu et al. [14] have explained the Approach of Expert System for Rainfall Prediction Based on Data Series. This strategy consolidates the Support Vector Machine (SVM) and Fuzzy Logic techniques. Predictive models keeping in mind the end goal to foresee downpour power (mm/day) in Athens, Greece, by Artificial Neural Networks (ANN) models, has been raised by P. T. Nastos et al. [15]. The ANNs results stress the conspired mean, greatest and least month to month downpour power for the following four progressive months in Athens. A Prediction Model by a method for Artificial Neural system has been connected by K. Abhishek et al. [16]. For the most phenomenal presentation, this report executed one of those capacities by building instructing and testing information sets and finding the quantity of disguised neurons in these layers. One of the principle issues with these strategies is that error is delivered when the quality and movement of precipitation change nonlinearly with time. They likewise showed that, since forecast of precipitation uses large number of learning patterns (precipitation designs), it requires a lot of calculating time to adequately lessen blunder. To overcome the difficulty present in the above techniques, in this work we introduce a novel hybridization approach for rainfall prediction.

\section{Data Collection and Analysis}

The study was directed by utilizing watched aggregate daily precipitation information got from two spots in Tamil Nadu. One of the spots is situated in pechiparai with pin code of 629161. The normal yearly precipitation in the catchment is 2,240 $\mathrm{mm}$ (88 in), and the normal yearly yield at the dam site is $218,000,000 \mathrm{~m} 3(7.7 \times 109 \mathrm{cu} \mathrm{ft})$. A direction of the dam is $8^{\circ} 23^{\prime} 4^{\prime \prime} \mathrm{N}$ and $77^{\circ} 22^{\prime} 28^{\prime \prime} \mathrm{E}$. Another spot is perunchani which is only $15 \mathrm{~km}$ far from the perunchani. The normal yearly precipitation in the catchment is $2,240 \mathrm{~mm}$ (88 in), and the normal yearly yield at the dam site is $218,000,000 \mathrm{~m} 3$ $(7.7 \times 109 \mathrm{cu} \mathrm{ft})$. A direction of DAM is $8^{\circ} 23^{\prime} 4^{\prime \prime} \mathrm{N}$ and $77^{\circ} 22^{\prime} 28^{\prime \prime} \mathrm{E}$. In 2015 , because of substantial downpour fall, the inflow into Perunchani dam was 223 cusecs and the release was 100 cusecs, while the water level of the dam remained at 74.77 feet as against its full limit of 77 feet. Here, a sum of 48 years of approved day by day precipitation information from January 1966 through December 2014 was gotten from Tamil Nadu State Meteorological Service. The information of February 29 was expelled for consistency. In the wake of breaking down perunchani information, the aggregate of 192 specimens was chosen to train RBF-HGSPSO; the aggregate of 96 specimens was chosen to test RBF-HGSPSO. Correspondingly, in pechiparai information, the aggregate of 392 specimens was chosen to train RBF-HGSPSO; the aggregate of 189 specimens was chosen to test RBFHGSPSO. Figure 1 demonstrates the normal month to month precipitation, assumed control over a period from 1966 to 2014, in Pechiparai and perunchani. In figure 1(a), one peak of precipitation amid a year in September. 


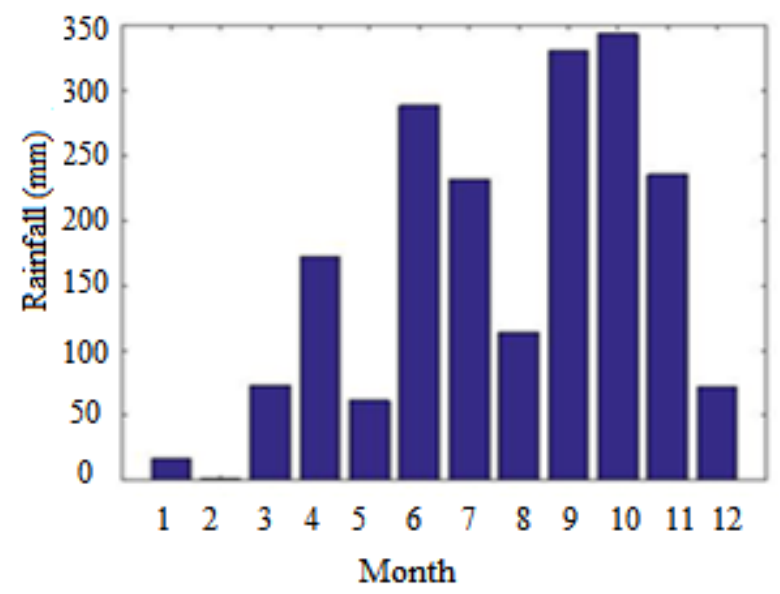

(a)

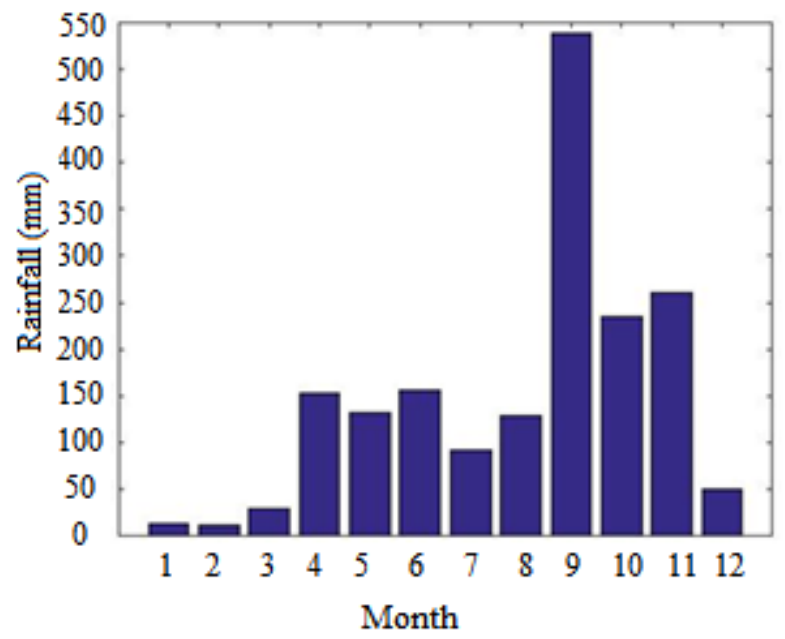

(b)

Figure.1 Average monthly rainfall (a) pechiparai and (b) Perunchani

\section{Proposed Rainfall Prediction Model}

The main goal of this research is to create a rainfall prediction model that can be used as a decision support tool to predict the future behavior by using five technical indicators and optimized RBF-NN. Our proposed work mainly consists of two phases such as (i) preprocessing based on feature indicator and (ii) prediction based on radial basis function neural network (RBF-NN).

\subsection{Preprocessing based on feature indicator}

At first, we consider the dataset $D^{D}$ which has $n$ periods of rainfall information. The preprocessing is the important stage for rainfall prediction. In this work, preprocess is done with the help of feature indicator. Our preprocessed data consists of five indicators along with corresponding class values. Here, the class value is well-defined for every single year that is organized on the basis of the subsequent year average of rainfall value. The Channel index
(CI), Ulcer index (UI), Rate of change (ROC), Relative strength index (RSI) and Average directional movement index (ADX) are the preprocessed four feature indicators. For all the experimentation with the rainfall data, the window size is constantly fixed as 7 which are decided based on the trial and error method. For the selection of best window size, the window size is changed from 5 to 10 and the output is computed. Based on the performance, the window is fixed as 7 for all the experimentation. Feature indicator description is detailed in the section below;

\section{(a) Channel index (CI)}

The present rainfall level in relation to an average rainfall level over a specified period of time is measured by Channel Index. For the calculations of the simple moving average and Mean Deviation the number of CI periods is also utilized. The channel index and typical rainfall values (TRV) are measured based on equation (1), (2).

$$
\begin{gathered}
C I=\frac{\left(\text { TRV }-7 \text { periodSMAof } T^{R}\right)}{(0.015 \times \text { Mean Deviation })} \\
\text { Typicalrain fall value }=\frac{\left(H^{R}+L^{R}+C^{R}\right)}{(3)}
\end{gathered}
$$

Where, High rainfall $\left(H^{R}\right)$ is the rainfall that is the highest rate at a specific period. Low rainfall $\left(L^{R}\right)$ is the rainfall that is the least amount rate at an exact period. The rainfall value at the end of the period is closing rainfall $\left(C^{R}\right)$. Closing rainfall plots offer a good perspective on the trending direction of the future rainfall level.

\section{* Computation of Simple moving average (SMA)}

For example, yearly closing rainfall value: 20,21,22,23,24,25,26,27,28,29,30,31,32

First year of 7-year SMA: $(20+21+22+23+$ $24+25+26) / 7=23$

Second year of 7-year SMA: $(21+22+23+24+$ $25+26+27$ ) $/ 7=24$

Seventh year of 7-year SMA: $(26+27+28+29+$ $30+31+32) / 7=29$

Constant $=0.015$

\section{* Computation of mean deviation}

Here, we used four steps to computing Mean Deviation:

- Primarily, subtract the most fresh 7-period average of the characteristic rainfall from typical rainfall value of all period. 
- Following that, yield the complete values of these numbers.

- Formerly, sum the complete values.

- Lastly, distribute it using the entire number of periods seven.

\section{(b) Ulcer index (UI)}

With the help of a default setting of 7 periods, the UI reproduces the predictable percentage drawdown over this period.

$$
\begin{array}{r}
R_{i}=\frac{\left(C^{R}\right)_{i}-\max C^{R}}{\max C^{R}} \\
\text { Ulcer index }=\sqrt{\frac{\sum_{i=1}^{N} R_{i}^{2}}{N}}
\end{array}
$$

Where; $\mathrm{N}$ is the time period of size or window $(N=7),\left(C^{R}\right)_{i}$ is the final rainfall in an exacting period, $R_{i}$ is the drawdown from the earlier high.

\section{(c) Rate of Change (ROC)}

The Rate of Change (ROC) indicator is simple, and it measures the percent change in rainfall from one period to the next. It is defined as the rainfall change in particular fixed time duration. The ROC calculation compares the current rainfall with the rainfall " $n$ " periods ago.

$$
\begin{aligned}
& \text { (Rainf allin lastmonthof window- } \\
& R O C=\frac{\text { last' } \left.n^{\prime} \text { peroidsago }\right)}{\text { last' } n^{\prime} \text { peroidsago }} \times 100
\end{aligned}
$$

\section{(d) Relative strength index (RSI)}

Relative strength index (RSI) fundamentally comprises of three constituents like; RS, average positive grow rainfall and average negative rainfall.

$$
\begin{gathered}
R S I=100-\frac{100}{1+R S} \\
R S=\frac{\text { Average positive grow rain fall }}{\text { Averagenegative grow rain fall }}
\end{gathered}
$$

The actual primary intentions for average positive grow rainfall (APGR) and also average negative grow rainfall (ANGR) are simple 7 period averages.

$$
\begin{gathered}
\text { First } A P G R=\frac{\text { Sumof PGR overthe past } 7 \text { peroids }}{7} \\
\text { First } A N G R=\frac{\text { Sumof } \text { NGR overthe past } 7 \text { peroids }}{7}
\end{gathered}
$$

The second, and following, computations are on the basis of the preceding averages and the present positive negative rainfall produces.

$$
\begin{aligned}
& A P G R=\frac{(\operatorname{Pr} \text { evious } A P G R) \times 6+\text { current positive rain flow }}{7} \\
& A N G R=\frac{(\operatorname{Pr} \text { eviousANGR }) \times 6+\text { currentnegativerain flow }}{7}
\end{aligned}
$$

\section{(e) Average directional movement index (ADX)}

The ADX indicator is an amalgamation of positive directional indicator or + DI and negative directional indicator or - DI. Together these are combined and the solution is leveled using an exponential moving average. Later Directional Index is computed with the help of dividing Directional Movement by True Range.

$$
\begin{aligned}
& \text { True range }=\max [(\text { high }- \text { low }) \text {, } \\
& \text { abs(high-close prev }) \text {, } \\
& a b s(\text { low-close } \text { prev })
\end{aligned}
$$

That is, Directional Indicator of day $1=$ Directional Movement of day 1 / True Range of day 1.

$$
\begin{aligned}
& +D I=\left(+D M_{1} / T R_{1}\right) \times 100 \\
& -D I=\left(-D M_{1} / T R_{1}\right) \times 100
\end{aligned}
$$

\section{2 predictions based on radial basis function neural network}

After preprocessing, we forecast the future rainfall on the basis of the Radial Basis Function Neural Network (RBFNN) [17].

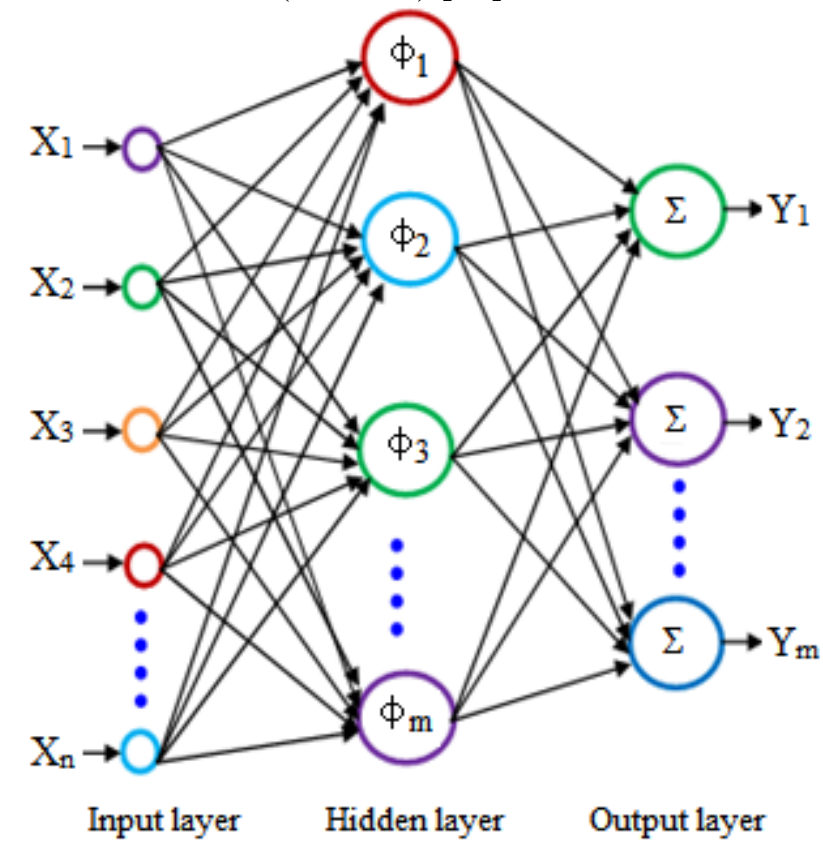

Figure.2 Working structure of RBF-neural network 
In this paper, an actual hybrid optimization strategy with the help of integrating the adaptive optimization of Grey Wolf optimization (GWO) into particle swarm optimization (PSO), specifically HGWPSO, is utilized for defining the parameters of radial basis performance neural networks (number of neurons, their corresponding centers, radii and weight) spontaneously. The basic architecture of a three-layered RBF-NN is shown in Figure 2. The network is normally collected of three layers: an input layer, a single unseen layer of nonlinear treating neurons, and an output layer. The output of the RBF-NN is designed best owing to

$$
\begin{aligned}
& Y^{s}=\sum_{i=1}^{N} W_{s i} h_{i}\left(x, o_{i}\right) \\
& =\sum_{i=1}^{N} W_{s i} h_{i}\left(\left\|x-o_{i}\right\|_{2}\right), \quad s=1,2, \ldots, m
\end{aligned}
$$

Where,

$$
x \in R^{n \times 1} \rightarrow \text { input vector }
$$

$h_{k}(.) \rightarrow$ radial basis function

$\|\cdot\|_{2} \rightarrow$ Euclidean norm

$W_{s i} \rightarrow$ Weights of output layer

$N \rightarrow$ Number of neuron

$O_{i} \rightarrow$ Center

A standardized Gaussian function characteristically is used as the radial basis performance as follows:

$$
h_{i}\left(x, c_{i}\right)=\exp \left(\frac{-\left\|x-c_{i}\right\|_{2}}{R_{i}^{2}}\right)
$$

In which $R_{i}$ indicate the radius of the $i^{\text {th }}$ node. It has been established that when enough units are distributed, a RBF-NN can vague any multivariate continuous performance as expected. In this article, a hybrid GWOPSO algorithm is utilized to discover the best constituents of the RBF-NN that imprecise a function on the board of a rainfall time sequence.

\subsubsection{Hybrid of GWO and PSO for RBF-NN design}

\section{Step 1: Population initialization}

The population initialization is the important stage in the entire optimization algorithm. Here, we utilized to launch the best constituents of the RBF$\mathrm{NN}$ that imprecise a function, comprising the number of unseen neurons, the centers $C_{i}$, radii $R_{i}$ and weights $W_{i}$. The unseen nodes are programmed as binary code string, 1 with association to input and output nodes and 0 with no connection.

\section{Step 2: Fitness calculation and ranking}

The selection of the fitness is a crucial aspect in HGWPSO algorithm. It is utilized to assess the aptitude (goodness) of candidate solutions. Here, Prediction error value is the main criteria used to design a fitness function. The fitness computation is executed for each solution. Suppose that we have a training set $T R=\left\{\left(x t, y_{t}\right), t=1,2, \ldots . m\right\}$, where, $y_{t}$ is the output. The fitness function has defined the equation (17). Evaluate the fitness of each of the $4 N$ individuals. Rank them on the basis of the fitness values.

$$
\begin{array}{r}
F i t=\min \left(E_{i}(x)\right) \\
E_{i}(x)=\frac{1}{m} \sum_{i=1}^{m}\left(y_{i}-o_{i}\right)
\end{array}
$$

Where,

$y_{i} \rightarrow$ Target output

$o_{i} \rightarrow$ Obtained output

\section{Step 3: Selection operation}

In every generation, afterwards the fitness values of all the persons in the similar population are considered, the top $2 \mathrm{~N}$ individuals are nominated. These $2 N$ individuals are known finest result and these are given to the GWO and PSO.

\section{Step 4: Apply GWO algorithm}

Grey wolf (Canis lupus) fits to Canidae family. Grey wolves are measured as apex predators, implication in which they are at the top most of the food chain. Grey wolves typically desire to living in a pack. The set size is 5-12 on average. To scientifically model the social hierarchy of wolves if designing GWO, we deliberate the fittest result as the alpha $\alpha$. The alpha $(\alpha)$ is respected as the most appropriate procedure with a view point to duplicating rationally the social pecking order of wolves though considering the GWO. Thus, the second and the third best arrangements are termed as beta $(\beta)$ and delta $(\delta)$ distinctly. The residual applicant arrangements are observed to be the omega $(\omega)$. The hunting (optimization) is directed with the help of the $\alpha, \beta, \delta$ and $\omega$ in the GWO technique.

\section{- Separate the solution based on fitness}

After fitness, calculation based on Eq. (17) 
separate the solution based on the fitness value. Let the first best fitness solutions be $F_{\alpha}$, the second best fitness solutions $F_{\beta}$ and the third best fitness solutions $F_{\delta}$.

\section{- Encircling prey}

The hunting is guided by $\alpha, \beta, \delta$ and $\omega$ trail these three candidates. In order for the pack to hunt a prey is first encircling it.

$$
\begin{gathered}
X(t+1)=X(t)-\vec{A} \cdot \vec{K} \\
\vec{K}=|\vec{C} . X(t+1)-X(t)| \\
\vec{A}=2 \vec{a} r_{1}-\vec{a} \text { And } \vec{C}=2 r_{2}
\end{gathered}
$$

Where, $\mathrm{t}$ is iteration number, $X(t)$ is prey position, A, C are coefficient vector, $\vec{a}$ is linearly decreased from 2 to 0 and $r_{1}, r_{2} \rightarrow$ Random vector [0, 1]

\section{- Hunting}

We undertake that the alpha (best candidate solution), beta and delta have the enhanced information about the potential site of the prey to replicate scientifically the hunting performance of the grey wolves. As a solution, we store the first three best results reached so far and need the other search agents (as well as the omegas) to study their positions permitting to the position of the best search agent. For recurrence, the novel solution $X(t+1)$ is assessed with the help of the formulae revealed as follows.

$$
\begin{gathered}
\vec{K}^{\alpha}=\left|\vec{C}_{1} \cdot F_{\alpha}-F\right|, \quad \vec{K}^{\beta}=\left|\vec{C}_{2} \cdot F_{\beta}-F\right|, \quad \vec{K}^{\delta}=\left|\vec{C}_{3} \cdot F_{\delta}-F\right| \\
F_{1}=F_{\alpha}-\vec{A}_{1} \cdot\left(\vec{K}^{\alpha}\right), F_{2}=F_{\beta}-\vec{A}_{2} \cdot\left(\vec{K}^{\beta}\right), F_{3}=F_{\delta}-\vec{A}_{3} \cdot\left(\vec{K}^{\delta}\right) \\
F(t+1)=\frac{F_{1}+F_{2}+F_{3}}{3}
\end{gathered}
$$

It can be perceived that the concluding position would be in a random place including a circle that is distinct using the points of alpha, beta, and delta in the search space. In added arguments alpha, beta, and delta evaluate the position of the prey, and other wolves informs their positions arbitrarily nearby the prey.

\section{- Attacking prey (exploitation) and Search for prey (exploration)}

Exploration and exploitation are failsafe with the help of the adaptive values of $b$ and $B$. The adaptive values of parameters $b$ and $B$ let GWO to effortlessly transition among exploration and exploitation. With declining $\mathrm{A}$, half of the repetitions are dedicated to exploration $(|B| \geq 1)$ and the rest half are devoted to exploitation $(|A|<1)$. The GWO has only two chief parameters to be accustomed ( $b$ and $C$ ). Though, we have retained the GWO algorithm as humble as likely with the smallest operators to be accustomed. The procedure will be sustained until the maximum number of iteration is gotten. Lastly the optimal solutions are designated on the basis of the fitness value.

\section{Step 5: Apply PSO algorithm}

After the GWO algorithm, then we apply PSO algorithm. Population-based search algorithm is known to be Particle swarm optimization (PSO). The velocity and position update are based on Eq. (25) and (26).

$$
\begin{gathered}
V_{i}^{d}=V_{i}^{d}+c_{1} \cdot \eta_{1} \cdot\left(p b_{i}^{d}-x_{i}^{d}\right)+c_{2} \cdot r_{2} \cdot\left(g b^{d}-x_{i}^{d}\right) \\
x_{i}^{d}=x_{i}^{d}+\delta V_{i}^{d}
\end{gathered}
$$

Where,

$c_{1}, c_{2}$ - constants with the value of 2.0

$r_{1}, r_{2}$ - independent random numbers generated in the range [0.1]

$V_{i}^{d}$ - Velocity of $i^{\text {th }}$ particle

$x_{i}^{d}$ - Current position of the particle $i$

$p b_{i}^{d}$ - Best fitness value of the particle at the current iteration

$g b^{d}$ - Best fitness value in the swarm.

\section{Step 6: hybridization}

After $n$ number of iteration completed; we hybrid these two algorithms based on the fitness of the corresponding solution. Here, we Compare GWO and PSO solution, if GWO fitness value $\left(\mathrm{GWO}_{\mathrm{i}}\right)$ is less than PSO fitness $\left(\mathrm{PSO}_{\mathrm{i}}\right)$ mean we consider the PSO solution. Else PSO fitness is less than the GWO means we consider the GWO solution.

\section{Step 7: Termination criteria}

The algorithm discontinues its execution only if a maximum number of iteration is achieved and the solution which is holding the best fitness value is selected and it is specified as best Parameters for prediction. Once the best fitness is attained by means of HGWPSO algorithm, selected solution is allocated for RBF neural network. 


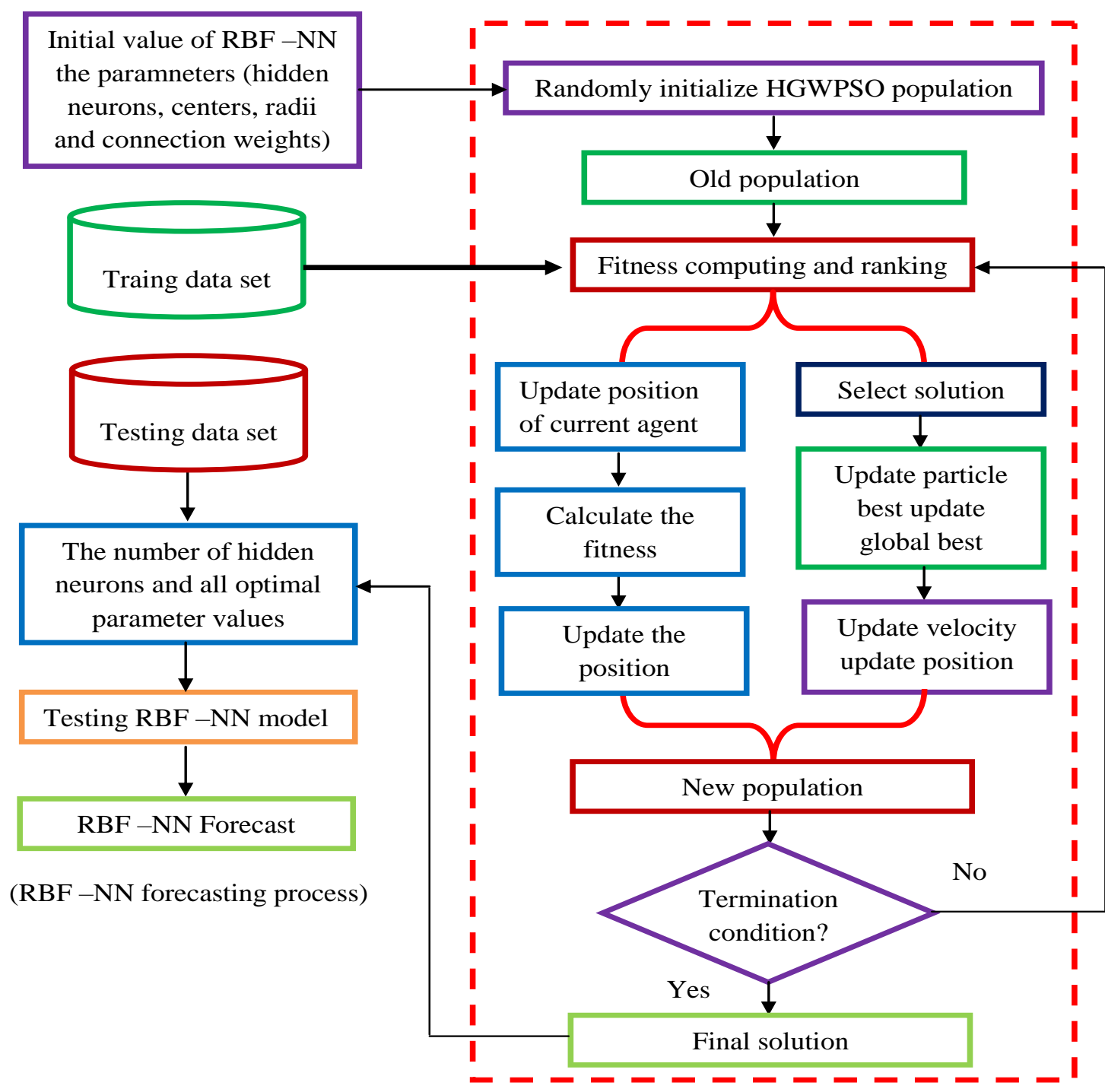

(Hybrid GWO -PSO optimization

Figure.3 Training and Testing process of proposed rainfall prediction system

\subsection{Testing phase}

In the testing phase, select the best solution from the training phase, which we have given to the input of the RBF-NN. Subsequently, we obtain the final rainfall value after the completed testing phase. The Training and Testing process of proposed rainfall prediction system are illustrated in figure 3 .

\section{Result and Discussion}

The platform adapted to develop the RBFHGWPSO approach is a PC with the following features: intel (R) core i5 processor, $3.20 \mathrm{GHz}, 4 \mathrm{~GB}$ RAM, a Windows 7 Operating system and the mat lab version 7.12 development environments. Moreover, this section explains the experimental results of proposed rainfall prediction technique using real datasets.

\subsection{Criteria for evaluating model performance}

Four different types of standard statistical performance evaluation criteria were employed to evaluate the performance of various models developed in this paper. These are Mean Absolute Percentage Error (MAPE), Mean squared error (MSE), mean absolute error (MAE) and Pearson Relative Coefficient (PR).

* Mean squared error (MSE)

$$
M S E=\sqrt{\frac{1}{N} \sum_{t=1}^{N}\left(P_{t}-D_{t}\right)}
$$

- Mean Absolute Percentage Error (MAPE)

$$
M A P E=\frac{1}{N} \sum_{t=1}^{N}\left|\frac{P_{t}-D_{t}}{P_{t}}\right|
$$

- Mean absolute error (MAE) 


$$
M A E=\frac{1}{N} \sum_{t=1}^{N}\left|P_{t}-D_{t}\right|
$$

* Pearson Relative Coefficient (PR)

$$
P R=\frac{\sum_{t=1}^{N}\left(P_{t}-\overline{P_{t}}\right)\left(D_{t}-\overline{D_{t}}\right)}{\sqrt{\sum_{t=1}^{n}\left(P_{t}-\overline{P_{t}}\right)^{2}} \sqrt{\sum_{t=1}^{N}\left(D_{t}-\overline{D_{t}}\right)^{2}}}
$$

Where, $P_{t}$ is an Expected value for period $t, D_{t}$ is an Actual value of period $t, \bar{P}_{t}$ is an Expected mean value, $\bar{D}_{t}$ is an Actual mean value and $N$ is a Total number of test data.

\subsection{Comparative analysis of Proposed Technique}

An efficient rainfall prediction technique is proposed to predict the future rainfall. Here, firstly the input rainfall dataset is converted into a suitable format for prediction. The obtained experimental results such as sample results after converting data with feature indicators are illustrated in table 1 . The performance of proposed rainfall prediction technique is evaluated in terms of the evaluation metrics value. Here, with the aid of the input training and testing dataset, the values of MAPE, MSE, MAE and PR are estimated. By analyzing the results, proposed technique is found to yield better performance. Table 2 to 3 show a comparative analysis of proposed against existing approach for two datasets.

Table 2 illustrates the fitting accuracy and efficiency of the model in terms of various evaluation indices for 189 testing samples. From the table 2, we can generally see that learning ability of RBF-HGWPSO outperforms the other four models under the same network input. Here, we compare our proposed work with $\mathrm{RBF}+\mathrm{GWO}, \mathrm{RBF}+\mathrm{PSO}$, $\mathrm{RBF}+\mathrm{GA}, \mathrm{RBF}$ and GABC+FFNN [18]. In [18], the author predicts the rainfall using feature indicator and optimal neural network. As a consequence, poor performance indices in terms of MAPE, RMSE, MAD, and MSE can be observed in RBF-GA model than other five models.

Table 1. Sample results after converting data with feature indicators (pechiparai)

\begin{tabular}{|c|c|c|c|c|c|c|}
\hline Year & RSI & ROC & CI & UI & ADX & Class \\
\hline 2005 & -0.75 & 33.33333 & -66.6667 & 53.03301 & 100 & 0.196621 \\
\hline 2006 & 13 & 99.0099 & 66.66667 & 0 & NaN & 0.03853 \\
\hline 2007 & 0 & 99.0099 & 66.66667 & 0 & 100 & 0.07252 \\
\hline 2008 & 26 & 99.0099 & 66.66667 & 0 & 100 & 0.075414 \\
\hline 2009 & 5.1 & 99.0099 & 66.66667 & 0 & 100 & 0.074837 \\
\hline 2005 & 0 & 99.0099 & $\mathrm{NaN}$ & 0 & $\mathrm{NaN}$ & 1 \\
\hline 2006 & 0 & 99.0099 & $\mathrm{NaN}$ & 0 & $\mathrm{NaN}$ & 1 \\
\hline 2007 & 0 & 99.0099 & 66.66667 & 0 & 100 & 0.079799 \\
\hline 2008 & 25.2 & 95.45455 & 66.66667 & 0 & 100 & 0.116963 \\
\hline 2009 & 0 & 99.0099 & 66.66667 & 0 & 100 & 0.105997 \\
\hline
\end{tabular}

Table 2. comparative analysis of proposed against existing for pechiparai dataset

\begin{tabular}{|c|c|c|c|c|}
\hline Approaches & MSE & MAPE & MAE & PR \\
\hline RBF+GWO & 0.053988 & 0.541203 & 0.041844 & 0.311752 \\
\hline RBF+PSO & 0.057003 & 0.694908 & 0.044375 & 0.202904 \\
\hline RBF+GA & 0.060802 & 1.101749 & 0.049748 & 0.032566 \\
\hline RBF & 0.079671 & 0.663017 & 0.035684 & 0.426819 \\
\hline GABC+FFNN[18] & 0.05148 & 0.60576 & 0.0435 & 0.5234 \\
\hline RBF+HGWPSO & 0.032581 & 0.531564 & 0.023541 & 0.792431 \\
\hline
\end{tabular}

Table 3. Comparative analysis of proposed against existing for perunchani dataset

\begin{tabular}{|c|c|c|c|c|}
\hline Approaches & MSE & MAPE & MAE & PR \\
\hline RBF+GWO & 0.061948 & 0.563382 & 0.034129 & 0.080879 \\
\hline RBF+PSO & 0.064497 & 0.591523 & 0.038758 & 0.070463 \\
\hline RBF+GA & 0.069697 & 0.903112 & 0.042183 & 0.084211 \\
\hline RBF & 0.057036 & 0.549994 & 0.040037 & 0.101193 \\
\hline GABC+FFNN[18] & 0.042251 & 0.051654 & 0.00975 & 0.7256 \\
\hline RBF+HGWPSO & 0.006646 & 0.032908 & 0.003016 & 0.99192 \\
\hline
\end{tabular}


Table 2 also shows that the performance of RBF-HGWPSO is the best in a case study for training and testing samples. From table 2, the proposed rainfall prediction model is obtain the results of MAPE $=0.531564, \mathrm{MSE}=0.032581$, $\mathrm{MAE}=0.023541$ and $\mathrm{PR}=0.792431$. Likewise, table 3 shows the Comparative analysis of proposed against existing for the perunchani dataset. From table 3 , the proposed rainfall prediction technique is $\mathrm{MSE}=0.006646, \mathrm{MAPE}=0.032908, \mathrm{MAE}=0.003016$ AND $p r=0.99192$. From the result, we understand the proposed RBF-HGWPSO method obtain better result compare to other five models. Figure 4 shows the prediction results of five different models for 189 testing samples of pechiparai dataset; we can see that the testing results of RBF-HGWPSO model are best in all models. Similarly, figure 5 shows the prediction results of five different models for 189 testing samples of perunchani dataset. From the results, we clearly understand our proposed approach is outperformed compare to other approaches.

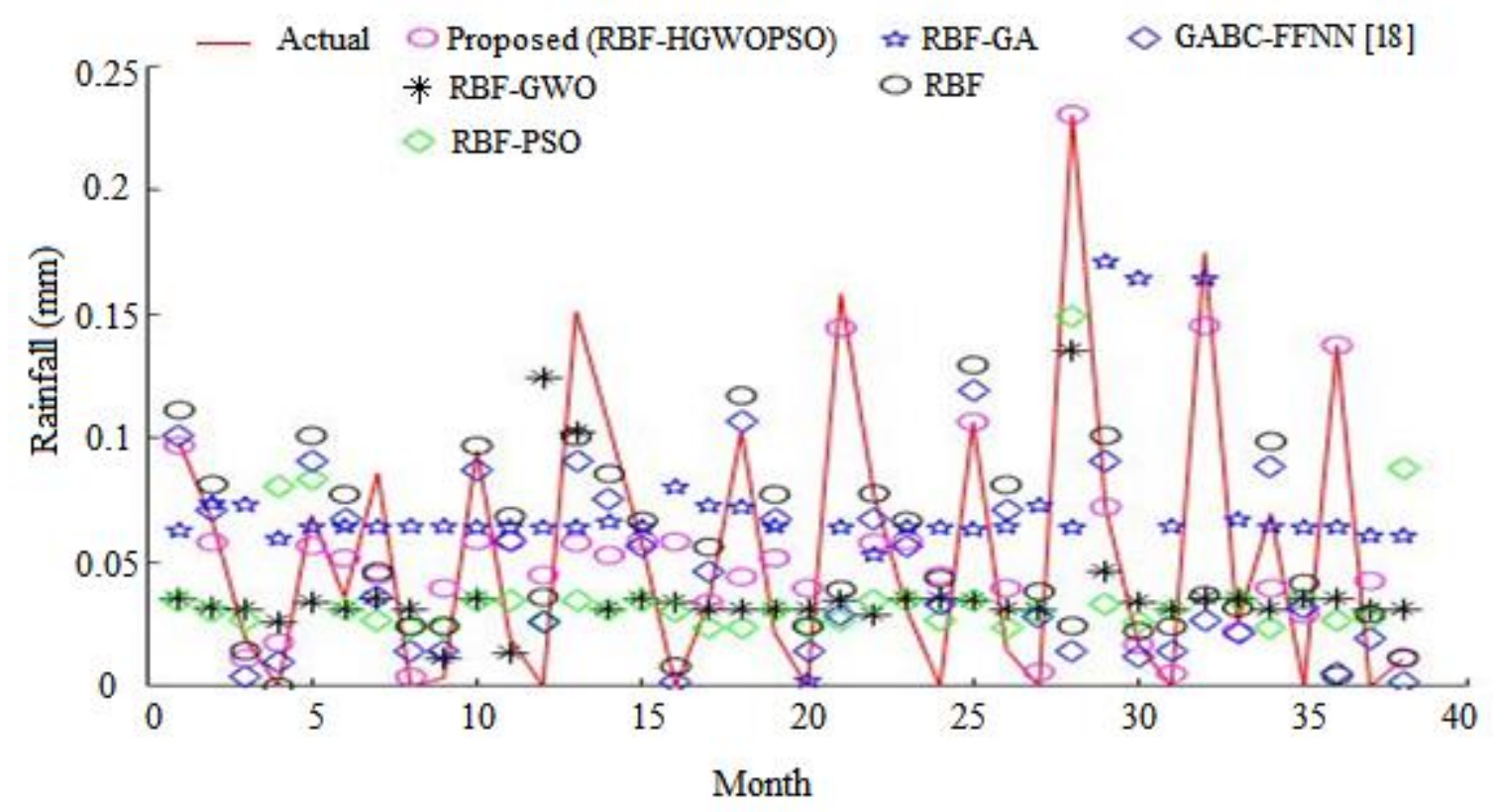

Figure.4 Prediction performances of five different models for 189 testing samples (pechiparai)

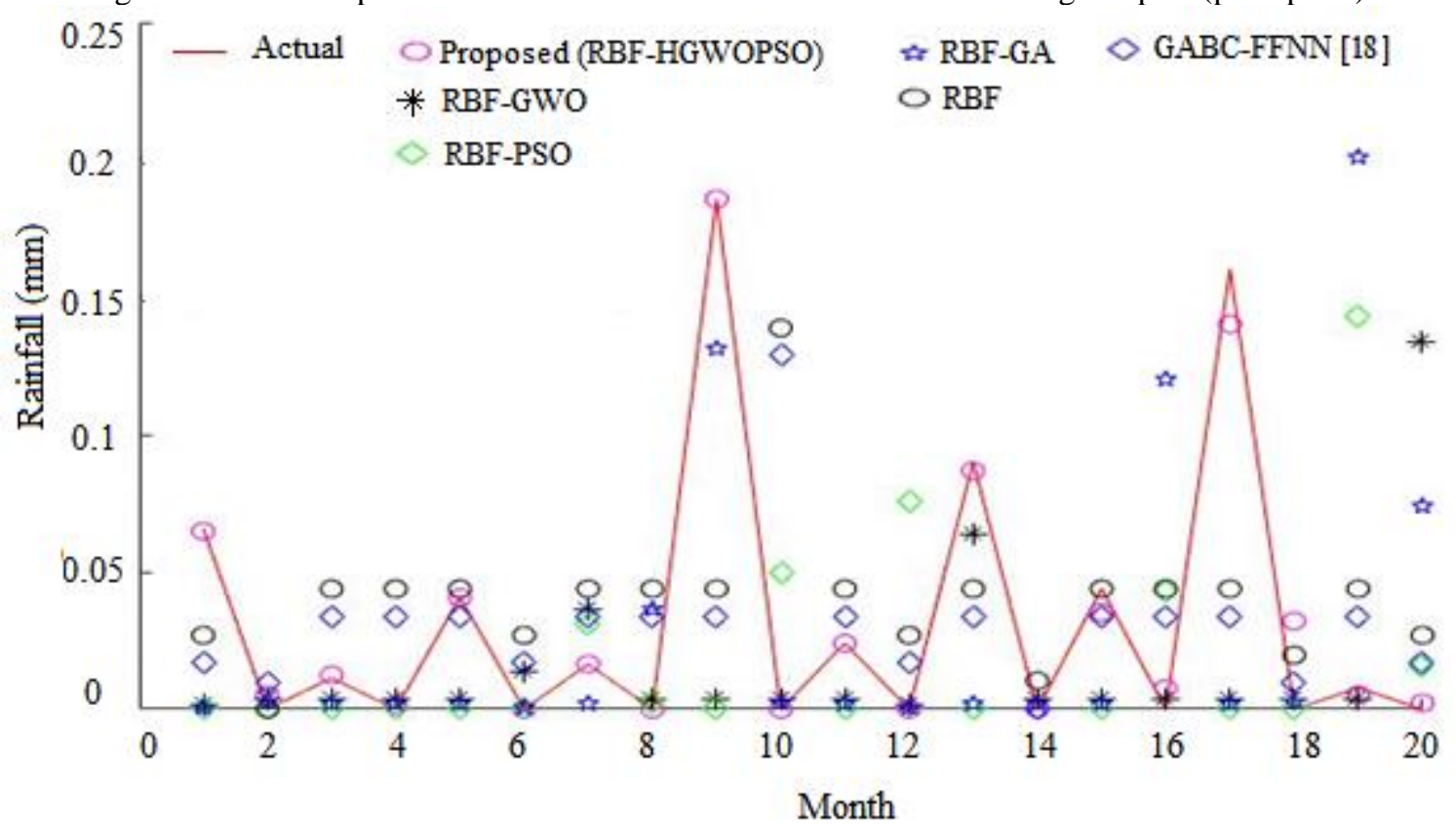

Figure.5 Prediction performances of five different models for 189 testing samples (Perunchani) 


\section{Conclusion}

In this paper, we have presented an efficient dissemination of rainfall forecasting to safeguard farmers from crop failure using optimized neural network model. Here, the proposed rainfall prediction consists of two phases, namely, preprocessing based on feature indicator and prediction based on radial basis function neural network (RBFNN). Firstly, the input rainfall dataset is preprocessed using feature indicators. There is five feature indicators used in the preprocessing step namely, channel index (CI), ulcer index (UI), rate of change (ROC), relative strength index (RSI) and average directional movement index (ADX). Subsequently, feature matrices are formed based on the preprocessed rainfall data. Once the feature matrix is formed, the prediction is done based on the hybrid classifier. In the hybrid classifier, GWO algorithm is combined with PSO for training the RBF neural network. The performance of the algorithm is analyzed with the help of real datasets taken from pechiparai, and perunchani regions in Tamil Nadu. Finally, from the comparative analysis, the proposed technique shows better results when compared other approaches. In future, we will used more parameters for predict the rainfall as well as we will predict other climate such as temperature and wind.

\section{References}

[1] M. Jajarmizadeh, S. Harun, R. Abdullah \& M. Salarpour, "Using soil and water assessment tool for flow simulation and assessment of sensitive parameters applying SUFI-2 algorithm”, Casp. J. Appl. Sci. Res. Vol. 2, No. 1, pp. 37-44, 2013.

[2] E. K. Lafdani, A. M. Nia, A. Pahlavanravi, A. Ahmadi \& M. Jajarmizadeh, "Daily rainfall-runoff prediction and simulation using ANN, ANFIS and conceptual hydrological", Int. J. of Engg Sci. and Tech, Vol. 1, No. 1, pp. 32-50, 2013.

[3] T. Hall, H. E. Brooks \& C. A. Doswell III, "Precipitation forecasting using a neural network", Weather and forecasting, Vol. 14, No. 3, pp. 338-345, 1999.

[4] D. Arvor, V. Dubreuil, J. Ronchail, M. Simões \& B. M. Funatsu, "Spatial patterns of rainfall regimes related to levels of double cropping agriculture systems in Mato Grosso (Brazil)", Int. J. of Climatology, Vol.34, No. 8, pp. 2622-2633, 2014.

[5] E. Toth, A. Brath \& A. Montanari, "Comparison of short-term rainfall prediction models for real-time flood forecasting", Journal of Hydrology, Vol. 239, No. 1, pp. 132-147, 2000.
[6] D. Broomhead \& D. D. Lowe, "Multivariable functional interpolation and adaptive networks", Journal of Complex System, Vol. 2, pp. 321-355, 1988.

[7] W. C. Hong, Rainfall forecasting by technological machine learning models, Journal of Applied Mathematics and Computation, Vol. 200, No. 1, pp. 41-57, 2008.

[8] J. Wu, "Prediction of rainfall time series using modular RBF neural network model coupled with SSA and PLS", In Asian Conference on Intelligent Information and Database Systems, Springer, Berlin Heidelberg, pp. 509-518, 2012.

[9] H. Turabieh, "A Hybrid ANN-GWO Algorithm for Prediction of Heart Disease", American Journal of Operations Research, Vol. 6, No. 2, pp. 136-146, 2016

[10] J. R. Zhang, J. Zhang, T. M. Lok and M. R. Lyu, "A hybrid particle swarm optimization-backpropagation algorithm for feed forward neural network training", Journal of Applied Mathematics and Computation, Vol. 185, No. 2, pp. 1026-1037, 2007.

[11]A. Altunkaynak and T. A. Nigussie, "Prediction of daily rainfall by a hybrid wavelet-season-neuro technique", Journal of Hydrology, Vol. 529, pp. 287301, 2015.

[12] J. Wu, J. Long and M. Liu, "Evolving RBF neural networks for rainfall prediction using hybrid particle swarm optimization and genetic algorithm", Journal of Neuro computing, Vol. 148, pp.136-142, 2015

[13] R. Hashim, C. Roy, S. Motamedi, S. Shamshirband, D. Petkovic, M. Gocic and S. C. Lee, "Selection of meteorological parameters affecting rainfall estimation using neuro-fuzzy computing methodology", Atmospheric Research, Vol. 171, pp. 21-30, 2016.

[14] Indrabayu, N. Harun, M. S. Pallu and A. Achmad, "A New Approach of Expert System for Rainfall Prediction Based on Data Series", International Journal of Engineering Research and Applications, Vol. 3, No. 2, pp. 1805-1809, 2013.

[15] K. Abhishek, A. Kumar, R. Ranjan, S. Kumar, "A Rainfall Prediction Model using Artificial Neural Network", IEEE Control and System Graduate Research Colloquium, pp. 82-87, 2012.

[16] P. T. Nastos, K. P. Moustris, I. K. Larissi, A. G. Paliatsos, "Rain intensity forecast using Artificial Neural Networks in Athens, Greece", Journal of Atmospheric Research, Vol. 119, pp. 153-160, 2013.

[17] I. Rojas, H. Pomares, J. L. Bernier, J. Ortega, B. Pino, F. J. Pelayo \& A. Prieto, "Time series analysis using normalized PG-RBF network with regression weights", Journal of Neuro computing, Vol. 42, No. 1, pp. 267-285, 2002.

[18] B. K. Rani, "Effective Features and Hybrid Classifier for Rainfall Prediction", Int. J. of Comp. Intell. Sys., Vol. 7, No. 5, pp. 937-951, 2014. 